To appear in November 4th issue of Nature

\title{
Multiple stellar populations in the globular cluster $\omega$ Centauri as tracers of a merger event
}

\author{
Y.-W. Lee ${ }^{\star}$, J.-M. Joo ${ }^{\star}$, Y.-J. Sohn ${ }^{\star}$, S.-C. Rey ${ }^{\star}$, H.-c. Lee ${ }^{\star} \&$ A. R. Walker ${ }^{\dagger}$ \\ ${ }^{\star}$ Center for Space Astrophysics, Yonsei University, Seoul 120-749, Korea \\ ${ }^{\dagger}$ National Optical Astronomy Observatories/Cerro Tololo Interamerican Observatory \\ (NOAO/CTIO), Casilla 603, La Serena, Chile
}

The discovery of the Sagittarius dwarf galaxy ${ }^{1}$, which is being tidally disrupted by and merging with the Milky Way, supports the view that the halo of the Galaxy has been built up at least partially by the accretion of similar dwarf systems. The Sagittarius dwarf contains several distinct populations of $\operatorname{stars}^{2,3}$, and includes M54 as its nucleus, which is the second most massive globular cluster associated with the Milky Way. The most massive globular cluster is $\omega$ Centauri, and here we report that $\omega$ Centauri also has several distinct stellar populations, as traced by red-giant-branch stars. The most metal-rich red-giant-branch stars are about 2 Gyr younger than the dominant metal-poor component, indicating that $\omega$ Centauri was enriched over this timescale. The presence of more than one epoch of star formation in a globular cluster is quite surprising, and suggests that $\omega$ Centauri was once part of a more massive system that merged with the Milky Way, as the Sagittarius dwarf galaxy is in the process of doing now. Mergers probably were much more frequent in the early history of the Galaxy and $\omega$ Centauri appears to be a relict of this era.

As part of our investigation of the luminosity-metallicity relation of the RR Lyrae stars in the globular cluster $\omega$ Cen, we have obtained $2 \mathrm{~K} B V$ CCD frames with the CTIO 0.9-m 
telescope that cover $40 \times 40 \operatorname{arcmin}^{2}$ in a $3 \times 3$ grid centred on the cluster, and covering out to approximately half the tidal radius. In total, 40 - 42 frames were taken in each filter and each field. The seeing was between 1.0 and 1.7 arcsec, and all of the observing nights (April 5 - 10 1996) were fully photometric. The $B$ and $V$ magnitudes of individual stars were measured with the point-spread-function (PSF)-fitting programs DAOPHOT II and ALLSTAR in the standard manner ${ }^{4}$. As a by-product of this investigation, we obtained high-quality homogeneous $B V$ colour-magnitude data for more than 130,000 stars in the field toward $\omega$ Cen, which represents the most extensive photometric survey to date for this cluster.

Figure 1 shows a $V$ versus $B-V$ colour-magnitude diagram (CMD) for stars in our programme field. We note the presence of several distinct red-giant-branches (RGB)s with a red, presumably metal-rich, sequence well separated from other bluer metal-poor ones. This feature was not evident in previous photometry ${ }^{5}$ with smaller sample sizes and larger photometric uncertainties. The radial distribution of the most metal-rich RGB stars is not significantly different from those of the metal-poor ones, which confirms that they belong to $\omega$ Cen. Note also that the red-clump that must be associated with the most metal-rich RGB is clearly apparent, partially overlapping the metal-poor RGBs. The presence of other interesting features on the CMD, such as the blue-tail phenomenon of the horizontal-branch (HB) and the blue straggler stars, illustrates the diversity of stellar populations in this cluster. The signature of field-star contamination is also evident, primarily as a swathe of stars with $0.3 \lesssim(B-V)_{o} \lesssim 1.2$. These stars will belong to the foreground galactic disk population.

In order to further investigate the discrete nature of the RGB, we have plotted in Figure 2 a histogram of the distribution of colour difference between each RGB star and the RGB fiducial of the most metal-poor component. The RGB stars are selected in a relatively narrow magnitude range $12.4<V<12.9$, so that the field-star and red-clump contamination is minimized, and which also avoids artificial mixing in the histogram stemming from metallicity dependence of the RGB slope. The presence of several distinct RGBs is confirmed, although the most metal-rich component is not as well distinguished as in Figure 1 because of the small sample size of such stars 
in this magnitude range.

Figure 3 shows our population models. These illustrate the relative age estimation from the location of the red clump associated with the most metal-rich RGB with respect to blue HB stars associated with the most metal-poor component. Panel (a) shows the case where all stars have the same age despite their different metallicities, which produces a red HB that is clearly much bluer than the observed red-clump confined within the two most extreme RGBs. Panel (b) shows our best match with the observed CMD in Figure 1, which suggests that the most metal-rich population in $\omega$ Cen is some 2 Gyr younger than the most metal-poor population in this system. This internal age-metallicity relation is clear evidence that $\omega$ Cen has enriched itself over this timescale.

The multimodal metallicity distribution function and the age-metallicity relation found in this study suggest that the protocluster of $\omega$ Cen was massive enough to undergo some self-enrichment and several early bursts of star formation. The relatively extended enrichment period of about 2 Gyr then indicates that the initial evolution of $\omega$ Cen occurred away from the dense central regions of the young Galaxy, for if this were not the case, one would expect the gaseous material to have been stripped from the cluster on a much shorter timescale ${ }^{8}$. The plausible resolution of this problem is that $\omega$ Cen has evolved within a dwarf-galaxy-sized gas-rich subsystem (the Searle and Zinn ${ }^{9}$ fragment) until it merged with and disrupted by our Galaxy some 2 Gyr after the formation of its first-generation metal-poor stars, leaving its core as today's globular cluster $\omega$ Cen. This is consistent with the fact that the multiple populations observed in $\omega$ Cen bear a strong resemblance to the Sagittarius dwarf system, where three distinct RGBs are identified with an internal age-metallicity relation that spans more than 3 Gyr (refs 2,3). Alternatively, it might be argued that a merger of several clusters could also create a composite object with multiple populations. The well defined peaks in the metallicity distribution function in Figure 2, however, require a merger of at least four clusters, and the possibility of this occurring may be extremely low. Furthermore, cluster mergers could only have occurred in dwarf galaxies with velocity dispersions much smaller than the Milky Way's ${ }^{10}$. Thus, even if $\omega$ Cen was formed by 
cluster mergers, it is probably still a relic of a dwarf galaxy subsequently accreted by our Galaxy. If our interpretation is correct, the case of $\omega$ Cen and that of the Sagittarius dwarf system would provide direct evidence for past and continuing accretion of protogalactic fragments, which suggest that similar accretion events may have continued throughout the history of Galactic formation. Extensive photometric surveys for other massive globular clusters, especially those with peculiar CMD morphology, such as M22 with broad RGB like $\omega$ Cen, and NGC 2808 with bimodal HB distribution, will undoubtedly help to reveal whether they also represent the relics of the Galaxy building blocks. 


\section{References}

1. Ibata, R. A., Gilmore, G. \& Irwin, M. J. A dwarf satellite galaxy in Sagittarius, Nature 370, 194-196 (1994).

2. Sarajedini, A. \& Layden, A. C. A photometric study of the globular cluster M54 and the Sagittarius dwarf galaxy: Evidence for three distinct populations, Astron. J. 109, 1086-1094 (1995).

3. Layden, A. C. \& Sarajedini, A. The globular cluster M54 and the star formation history of the Sagittarius dwarf galaxy, Astrophys. J. 486, L107-L110 (1997).

4. Stetson, P. B. DAOPHOT: A computer program for crowded-field stellar photometry, Publ. Astron. Soc. Pacif. 99, 191-222 (1987).

5. Woolley, R. v. d. R. Studies of the globular cluster $\omega$ Centauri. I. Catalogue of magnitudes and proper motions, Royal Observatory Annals No. 2, 1-128 (1966).

6. Demarque, P. et al. New Yale isochrones (Yale University Observatory, New Haven (1996).

7. Lee, Y.-W., Demarque, P. \& Zinn, R. The horizontal-branch stars in globular clusters. II. The second parameter phenomenon, Astrophys. J. 423, 248-265 (1994).

8. Norris, J. E., Freeman, K. C. \& Mighell, K. J. The giant branch of $\omega$ Centauri. V. The calcium abundance distribution, Astrophys. J. 462, 241-254 (1996).

9. Searle, L. \& Zinn, R. Compositions of halo clusters and the formation of the galactic halo, Astrophys. J. 225, 357-379 (1978).

10. van den Bergh, S. Mergers of Globular Clusters, Astrophys. J. 471, L31-L32 (1996). 
Acknowledgements. Support for this work was provided by the Creative Research Initiatives Program of the Korean Ministry of Science \& Technology, and in part, by the Korea Science \& Engineering Foundation. S.-C.R. was a visiting astronomer at CTIO/NOAO, which is operated by the Association of Universities for Research in Astronomy, Inc., under cooperative agreement with the National Science Foundation.

Correspondence should be addressed to Y.-W. L. (e-mail: ywlee@csa.yonsei.ac.kr). 
Fig. 1.- Colour-magnitude diagram of 50,129 stars in the direction of $\omega$ Cen. These diagrams were obtained from a mosaic of nine $2 \mathrm{~K}$ CCD fields. Only stars with at least 20 detections and small photometric errors $\left(\sigma_{V}<0.05 \mathrm{mag}\right.$ and $\left.\sigma_{B-V}<0.071 \mathrm{mag}\right)$ have been plotted. Panel (a) is for all stars in our programme field, while panel (b) is only for stars located between $2.58^{\prime}$ and $15.48^{\prime}$ from the cluster centre. There are several distinct RGBs and a red-clump associated with the most metal-rich component. Two RGB loci from the new Yale isochrones ${ }^{6}(\mathrm{Z}=0.0004,0.005)$ are also compared in panel (b) that bracket the metallicity range of $\omega$ Cen.

Fig. 2.- Histogram of the distribution of colour difference. Colour difference between each RGB star and the RGB fiducial of the most metal-poor component, $\Delta(B-V)$, is plotted in the range $12.4<V<12.9$. The metallicities (Z values) of four distinct RGBs are also marked.

Fig. 3. - Stellar population models. This models illustrate the estimation of age difference between the red-clump associated with the most metal-rich $(\mathrm{Z}=0.005) \mathrm{RGB}$ and the blue HB associated with the most metal-poor $(\mathrm{Z}=0.0004)$ component. Dots and crosses are individual HB stars from synthetic HB models ${ }^{7}$ and the solid lines are from new Yale isochrones ${ }^{6}$. Panel (a) is for the case that all stars have the same age despite their different metallicities, while panel (b) is for the case that the most metal-rich population is 2 Gyr younger than the most metal-poor population. Only $\Delta t$ of about 2 Gyr reproduces the features on the observed CMD in Fig. 1. 


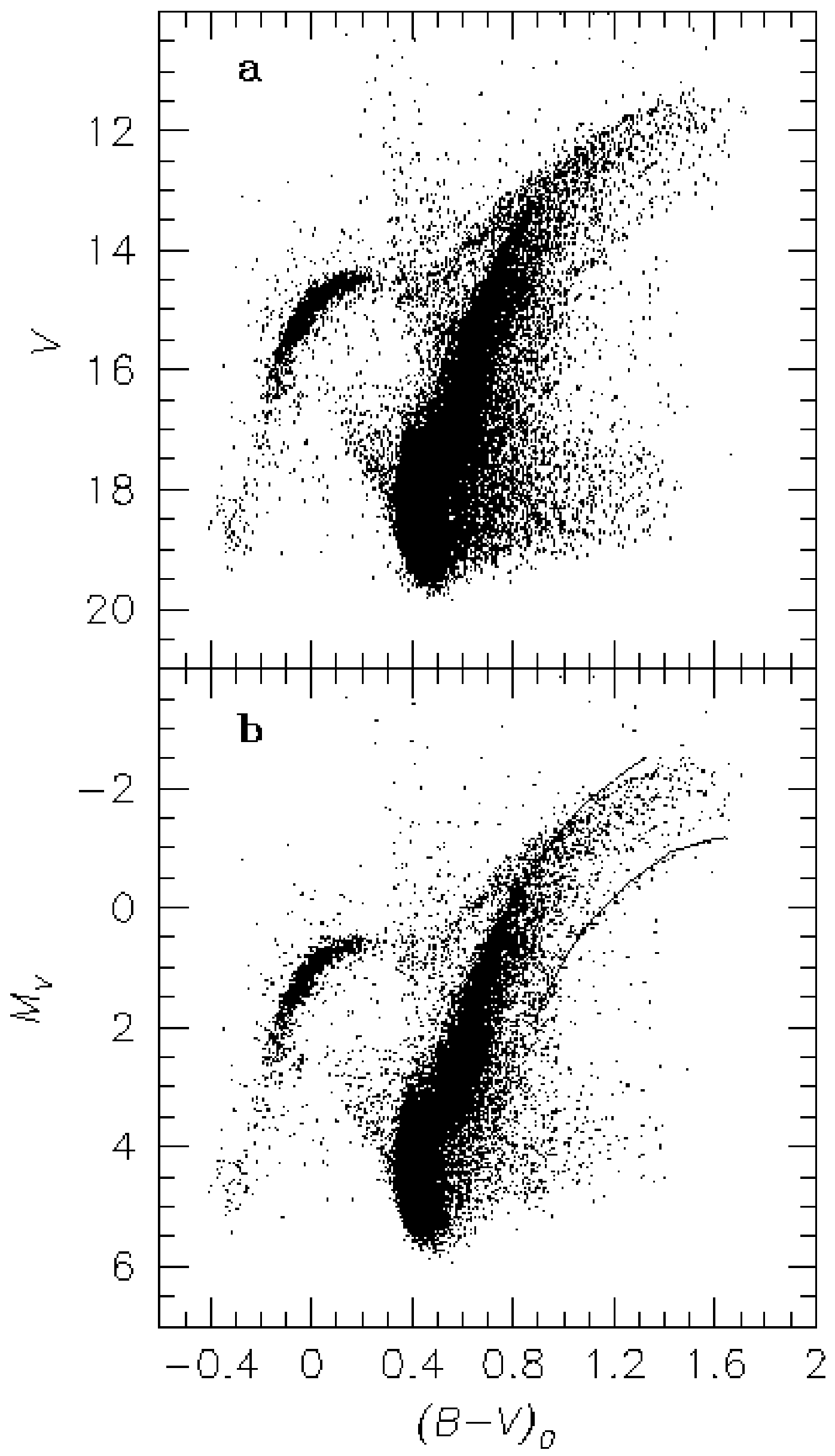




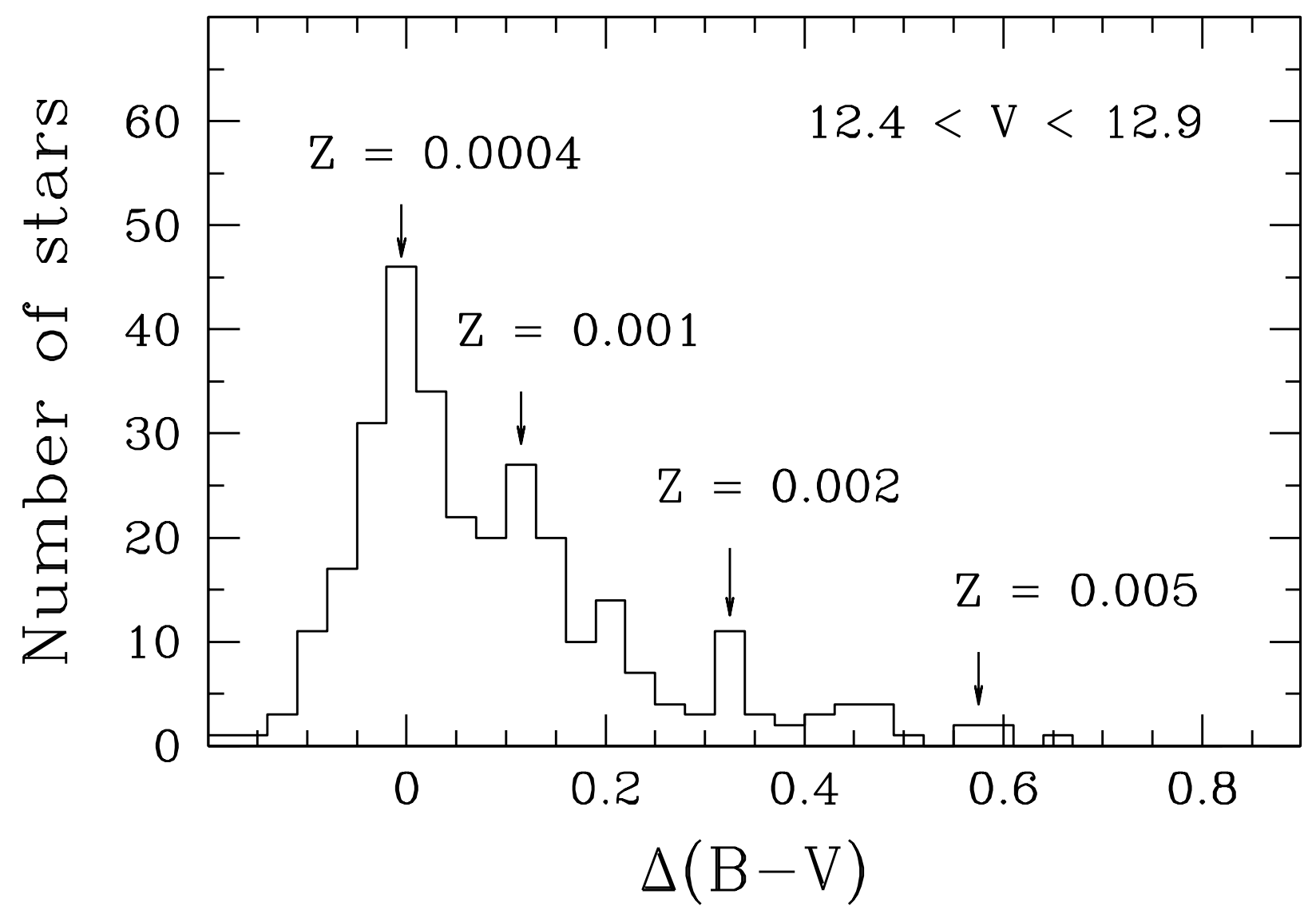




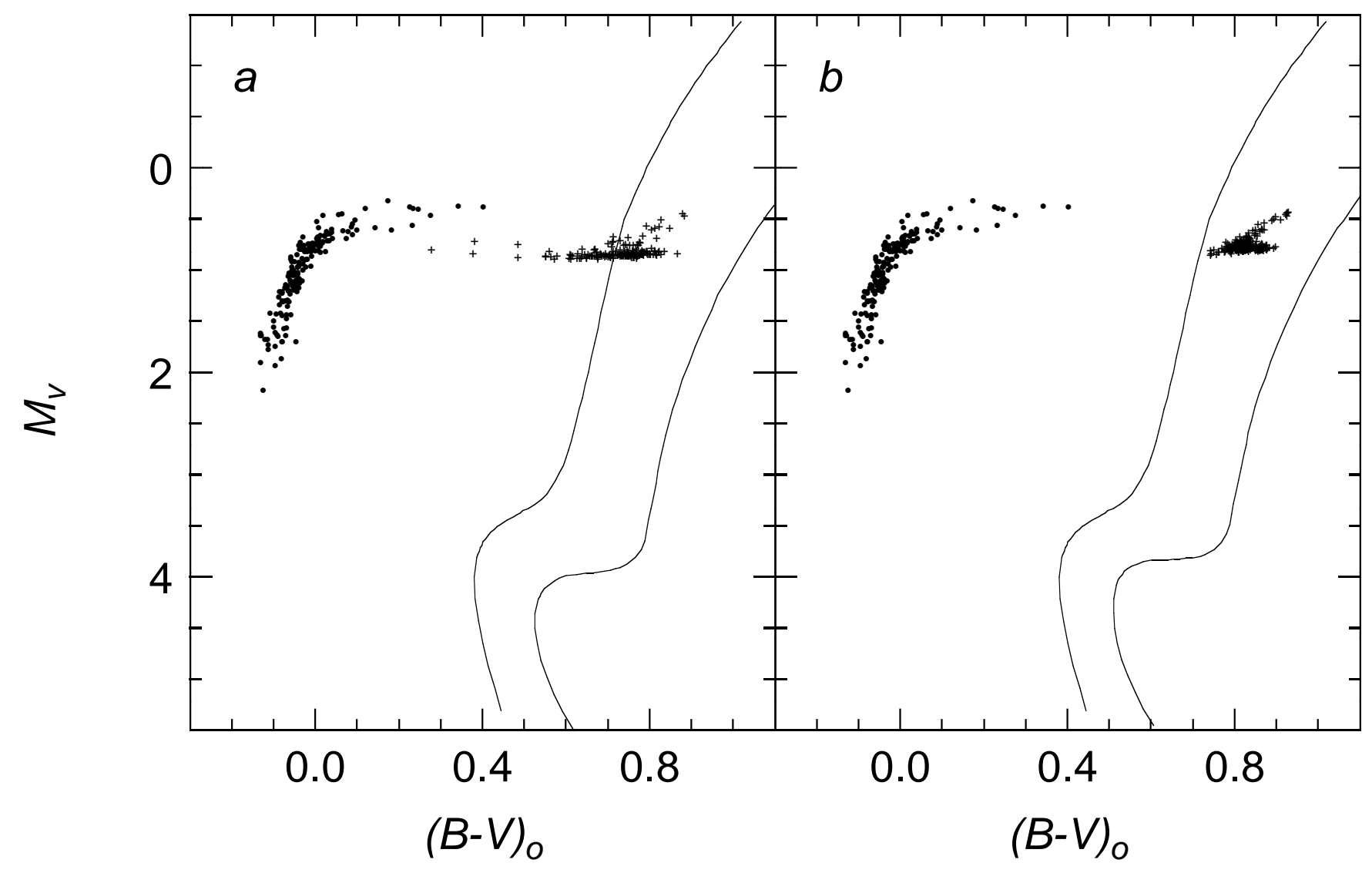

